\title{
Predictors of survival in critically ill patients with acute respiratory distress syndrome (ARDS): an observational study
}

\author{
Felix Balzer ${ }^{1}$ D, Mario Menk¹, Jannis Ziegler², Christian Pille ${ }^{1}$, Klaus-Dieter Wernecke $^{3}$, Claudia Spies ${ }^{1}$, \\ Maren Schmidt ${ }^{4}$, Steffen Weber-Carstens ${ }^{1 *+}$ and Maria Deja ${ }^{1,2^{*+}}$
}

\begin{abstract}
Background: Currently there is no ARDS definition or classification system that allows optimal prediction of mortality in ARDS patients. This study aimed to examine the predictive values of the AECC and Berlin definitions, as well as clinical and respiratory parameters obtained at onset of ARDS and in the course of the first seven consecutive days.

Methods: The observational study was conducted at a 14-bed intensive care unit specialized on treatment of ARDS. Predictive validity of the AECC and Berlin definitions as well as $\mathrm{P}_{2} \mathrm{O}_{2} / \mathrm{F}_{\mathrm{i}} \mathrm{O}_{2}$ and $\mathrm{F}_{\mathrm{i}} \mathrm{O}_{2} / \mathrm{P}_{2} \mathrm{O}_{2}{ }^{*} \mathrm{P}_{\text {mean }}$ (oxygenation index) on mortality of ARDS patients was assessed and statistically compared.

Results: Four hundred forty two critically-ill patients admitted for ARDS were analysed. Multivariate Cox regression indicated that the oxygenation index was the most accurate parameter for mortality prediction. The third day after ARDS criteria were met at our hospital was found to represent the best compromise between earliness and accuracy of prognosis of mortality regarding the time of assessment. An oxygenation index of 15 or greater was associated with higher mortality, longer length of stay in ICU and hospital and longer duration of mechanical ventilation. In addition, non-survivors had a significantly longer length of stay and duration of mechanical ventilation in referring hospitals before admitted to the national reference centre than survivors.

Conclusions: The oxygenation index is suggested to be the most suitable parameter to predict mortality in ARDS, preferably assessed on day 3 after admission to a specialized centre. Patients might benefit when transferred to specialized ICU centres as soon as possible for further treatment.
\end{abstract}

Keywords: Acute respiratory distress syndrome, Pao2/FIO2 ratio, Oxygenation index, Classification, Risk stratification, Outcome

\section{Background}

The American-European Consensus Conference (AECC) definition was commonly used by clinicians to categorize ARDS patients [1]. But issues regarding reliability of various criteria have emerged, including a poor interobserver reliability of chest radiograph interpretation, confusing acute lung injury (ALI) /ARDS nomenclature and the inconsistency of $\mathrm{PaO} 2 / \mathrm{FiO} 2$ ratio due to the effect of positive end-expiratory

\footnotetext{
* Correspondence: steffen.weber-carstens@charite.de; maria.deja@charite.de ${ }^{\dagger}$ Equal contributors

${ }^{1}$ Department of Anesthesiology and Intensive Care Medicine, Charité Universitätsmedizin Berlin, Campus Virchow-Klinikum / Campus Charité Mitte, Augustenburger Platz 1, D-13353 Berlin, Germany

Full list of author information is available at the end of the article
}

pressure (PEEP) [2]. Those limitations have recently been tackled with the establishment of the Berlin Definition in 2012 [3]. Herein, ARDS patients are classified into three independent categories (i.e., mild, moderate and severe ARDS) and additional variables are taken into account. Several factors, such as severity of chest radiograph, a PEEP level above $5 \mathrm{~cm}$ $\mathrm{H}_{2} \mathrm{O}$, low compliance and poor oxygenation, are now used to define severe ARDS.

The Berlin Definition addresses and clarifies some of the limitations of the AECC definition and is the first to include minimum ventilator settings. The predictive validity for mortality is only slightly better than in the AECC definition. However, it was not designed to serve 
as a prognostication tool [2]. In the past, other variables of interest, such as the $\mathrm{PaO} 2 / \mathrm{FiO} 2$ ratio, the oxygenation index (OI), the influence of co-morbidities and a number of clinical scores (SAPS, SOFA), were screened in ARDS patients to prompt early prediction of outcome and to ensure more efficient resource allocation [4-6]. As the ARDS is, however, a very heterogeneous syndrome with several different causes, all proposed definitions, parameters and variables did not resolve the problem adequately. For a reliable prediction of outcome and mortality in ARDS patients, comparable treatment strategies in respective hospitals are required. However, treatment algorithms applied on ARDS patients may differ significantly between different hospitals (usage of inhalative nitric oxide, ventilator settings, criteria to start lung assist devices) representing different approaches to the "state of the art" in ARDS therapy. These problems may be partly resolved in unicentric studies in specialized hospitals involving sufficiently high numbers of patients.

Outcome prediction in critically ill patients at a given point of time plays a major role (e.g. for appropriate treatment decisions and family communication). In this context, we examined the predictive values of the AECC and Berlin definition and also assessed alternative clinical parameters that are available in routine patient care.

\section{Methods}

This observational analysis was conducted at a 14-bed intensive care unit (ICU) of a national reference centre specialized on treatment of ARDS in adult patients with severely compromised medical conditions. On average, two thirds of all ARDS patients are transferred from other hospitals. Patients at our institution were treated according to a strong treatment algorithm $[7,8]$.

After written consent of the Ethics Commission at Charité - Universitätsmedizin Berlin (EA1/223/12), clinical routine data from all patients admitted for ARDS between January 2007 and December 2013 were extracted from the two electronic patient data management systems operated at the hospital (COPRA, Sasbachwalden, Germany and SAP, Walldorf, Germany). In addition to basic demographic data, we assessed length of stay and duration of prior mechanical ventilation in referring institutions, comorbidities (using Charlson comorbidity score [9]), ICU admission scores, and use of extracorporeal oxygenation in order to characterize the patient population. As major clinical causes leading to ARDS, we differentiated pneumonia, sepsis of extra-pulmonary origin, trauma, immunedeficiency and "acute on chronic", i.e. patients with an acute pulmonary disease on pre-existing chronic pulmonary disease (e.g.primary lung fibrosis, COPD $>/=$ GOLD 4 or cystic fibrosis), because these are well known influencing factors of mortality in ARDS patients [10, 11].

Day 1 of study inclusion was defined as the first day with a median $\mathrm{P}_{\mathrm{a}} \mathrm{O}_{2} / \mathrm{F}_{\mathrm{i}} \mathrm{O}_{2}$ below 300 at our hospital. Patient-specific data that was extracted on a daily basis comprised SOFA score, ventilator settings / respiratory parameters (tidal volume $\left(\mathrm{V}_{\mathrm{T}}\right)$, tidal volume / predicted body weight $\left(\mathrm{V}_{\mathrm{T}} / \mathrm{PBW}\right), \mathrm{P}_{\text {mean }}, \mathrm{P}_{\text {peak }}$, PEEP, static compliance, $\mathrm{F}_{\mathrm{i}} \mathrm{O}_{2}$ ), gas exchange using arterial blood gas analyses $\left(\mathrm{pH}, \mathrm{P}_{\mathrm{a}} \mathrm{O}_{2}, \mathrm{P}_{\mathrm{a}} \mathrm{CO}_{2}, \mathrm{P}_{\mathrm{a}} \mathrm{O}_{2} / \mathrm{F}_{\mathrm{i}} \mathrm{O}_{2}\right)$, use of nitric oxide and positioning therapy. Status of ARDS was assessed according to the definition of AECC [1] and the Berlin definition [3] upon admission on our ICU.

For analysing data based on ventilator settings and arterial blood gas analyses, the following algorithm was applied: Each day was divided in four intervals of six hours each. In each interval, the combination of ventilator settings and results of blood gas analyses with the least difference in time was chosen. For each of these parameters, the median was calculated and transferred to the study database. Ventilator settings had been saved approximately every $30 \mathrm{~min}$ in the electronic patient records and were only considered when they were documented prior to lab results.

Predictive validity for the AECC and Berlin definition as well as for $\mathrm{P}_{\mathrm{a}} \mathrm{O}_{2} / \mathrm{F}_{\mathrm{i}} \mathrm{O}_{2}$ and $\mathrm{F}_{\mathrm{i}} \mathrm{O}_{2} / \mathrm{P}_{\mathrm{a}} \mathrm{O}_{2}{ }^{*} \mathrm{P}_{\text {mean }}(\mathrm{OI})$ regarding mortality was assessed with receiver operator curves (ROC) and corresponding results for area under the curve (AUC). Kaplan-Meier curves were used to illustrate differences in survival using these four mentioned parameters. In order to show differences for continuous variables (i.e. $\mathrm{P}_{\mathrm{a}} \mathrm{O}_{2} / \mathrm{F}_{\mathrm{i}} \mathrm{O}_{2}$ and $\mathrm{F}_{\mathrm{i}} \mathrm{O}_{2} / \mathrm{P}_{\mathrm{a}} \mathrm{O}_{2}{ }^{*} \mathrm{P}_{\text {mean }}$ ), we selected the value that maximized the vertical distance between ROC curve and diagonal line (highest sum of sensitivity and specificity) [12]. This cut-off value was used to attribute patients to one of two groups (i.e. above or below calculated cut-off) in order to analyse predictive validity.

Descriptive analyses and statistical testing were performed using the $\mathrm{R}$ Project of Statistical Computing 3.0.1 with a $p$ value below 0.05 regarded as significant. When normal distribution was ruled out using the Kolmogorov-Smirnov test, results were given in median and interquartile range (IQR), otherwise mean \pm standard deviation (SD). Qualitative observations were characterized by numbers with percentage. Statistical significance among groups was univariately analyzed by the exact nonparametric Kruskal-Wallis-test and (pairwise) with the exact Mann-Whitney $U$ test. Exact Chi-Square tests were used for qualitative data. In order to test multivariately for influencing factors of mortality and survival, Cox regression was applied with stepwise backwards selection including variables that showed a statistically significant impact in univariate analyses. All tests should be 
understood as constituting explorative analysis, such as no adjustment for multiple testing has been made.

\section{Results}

The population analysed in this study comprised 442 critically-ill patients admitted for ARDS. As reflected by a median APACHE II admission score of 28 [20;35], a SAPS II admission score of 54 [39;70], a SOFA admission score of 12 [9;15] and a Charlson comorbidity index (CCI) of 3 [2-5], the study population was characterised by severe medical conditions (see Table 1). Along the line, patients required in median a PEEP of $17[15 ; 20]$ $\mathrm{cmH}_{2} \mathrm{Obar}, \mathrm{P}_{\text {mean }}$ of $25[21 ; 29] \mathrm{cmH}_{2} \mathrm{O}$, and $\mathrm{P}_{\text {peak }}$ of 36 [32;39] $\mathrm{cmH}_{2} \mathrm{O}$ on day 1 of protocol. Further respiratory parameters are shown in Additional file 1: Table S1 in the electronic supplement, indicating more invasive ventilation in non-survivors. In $89.3 \%$ of all patients, prone position was applied at least once within the first three days of protocol (see Additional file 2: Table S3 for further details). Non-survivors were significantly older than survivors. Also, lengths of stay in referring institutions as well as lengths of prior mechanical ventilation were longer in non-survivors. Although the aetiology of ARDS was overall not statistically different between survivors and non-survivors, the number of patients with acute on chronic respiratory failure was higher in non-survivors; trauma and sepsis of extrapulmonary origin were more frequent in survivors. Scores for description of severity of illness on ICU admission at our centre were significantly higher in the group of non-survivors. Extracorporeal lung assist devices (ELAD) including extracorporeal membrane oxygenation (ECMO) and extracorporeal lung assist (ECLA) were applied in 256 patients (57.9\%). When ELAD was required in patient, it was set up within the first three days of protocol in $87.5 \%$ (see Additional file 3: Table S4 for further details). Overall patient survival with ELAD was $43.0 \%$.

On day 1 of the protocol, 99 patients (22.4\%) presented with a $\mathrm{P}_{\mathrm{a}} \mathrm{O}_{2} / \mathrm{F}_{\mathrm{i}} \mathrm{O}_{2}$ between 200 and 300 , which corresponds to the stage of acute lung injury (ALI) in the AECC definition. Respectively, $\mathrm{P}_{\mathrm{a}} \mathrm{O}_{2} / \mathrm{F}_{\mathrm{i}} \mathrm{O}_{2}$ was below 200 in the remaining 343 patients (77.6\%). Applying the three stages of the Berlin definition, this corresponds to 99 patients (22.4\%) with mild, 210 patients $(47.5 \%)$ with moderate and 133 patients $(30.1 \%)$ with severe ARDS. The median for $\mathrm{P}_{\mathrm{a}} \mathrm{O}_{2} /$ $\mathrm{F}_{\mathrm{i}} \mathrm{O}_{2}(n=411)$ was 137 [93;193] and 16.9 [11.6;27.4] for OI $(n=391)$.

The predictive validity for in-hospital mortality of the four parameters mentioned above - AECC definition, Berlin definition, $\mathrm{P}_{\mathrm{a}} \mathrm{O}_{2} / \mathrm{F}_{\mathrm{i}} \mathrm{O}_{2}$ and $\mathrm{OI}-$ was calculated for the first seven days on ICU (see Fig. 1). In general, the area under the curve was lowest for all four parameters on day 1 and highest on day 7. Given that we aimed to determine the earliest possible day for outcome prediction and that all parameters showed a monotonous increase until day 3 , we decided to use clinical variables from that day for further analyses. As extracorporeal oxygenation was expected to have an impact on respiratory variables, sub-analyses were conducted regarding the predictive value of OI in patients with ELAD, without ELAD and in the entire patient population. As prognostic validity was highest in the group comprising all patients, we decided to evaluate all four categorizing variables regardless of possible extracorporeal oxygenation that might have been in place (see Additional file 4: Figure S1).

On day 3 of protocol, 31 patients had a $\mathrm{PaO}_{2} /$ $\mathrm{FiO}_{2}>300 \mathrm{mmHg}$. 32 patients died until day 3. Including data of all patients, resulting groups of all four classifications - AECC, Berlin definition, $\mathrm{P}_{\mathrm{a}} \mathrm{O}_{2} / \mathrm{F}_{\mathrm{i}} \mathrm{O}_{2}$ and OI - based on data from day 3 were depicted as Kaplan-Meier-curves in regards to in-hospital survival (Fig. 2). For the continuous parameters $\mathrm{P}_{\mathrm{a}} \mathrm{O}_{2} / \mathrm{F}_{\mathrm{i}} \mathrm{O}_{2}$ and $\mathrm{OI}$, groups were identified by calculating the cut-off values (137 for $\mathrm{P}_{\mathrm{a}} \mathrm{O}_{2} / \mathrm{F}_{\mathrm{i}} \mathrm{O}_{2}$ and 15 for OI respectively) distinguishing between survival or death according to the Youden method described above. Resulting curves in each of the four Kaplan-Meier graphs were significantly different $\left(\mathrm{p}_{\mathrm{Log} \text { rank }}<0.001\right)$ from a univariate perspective. Multivariate regression analyses indicated that not a singular parameter may be considered for reliable mortality prediction (see Additional file 5: Table S2). Hence, stepwise backwards selection allowed the identification of clinically valid combinations of explanatory variables. In the resulting model, OI was the only one of the four investigated categorizing variables that remained significant (HR 1.03, $95 \%$ CI 1.015-1.047, $p<0.001)$. With every one-point increase of OI, the risk of in-hospital death will increase by $3 \%$, whereas the risk of in-hospital death would increase by $36 \%$ if the OI increased by 10 points. Use of extracorporeal lung assist devices did not prove to be an independent predictor (Table 2).

OI - being an independent predictor in the final model of regression analysis - was then used to group patients concerning outcome criteria. Patients with an OI above the respective cut-off of 15 on day 3 had longer length of stay on ICU, longer length of hospital stay, and longer duration of mechanical ventilation. Furthermore, mortality was significantly higher, with patients less likely to be discharged to home or another facility (see Table 3).

\section{Discussion}

In this study, we analysed early predictive values for mortality of the AECC- and Berlin definition of ARDS, $\mathrm{P}_{\mathrm{a}} \mathrm{O}_{2} / \mathrm{F}_{\mathrm{i}} \mathrm{O}_{2}$ and oxygenation index in critically ill ARDS patients at the reference centre of the Charité Berlin. 
Table 1 Patient characteristics and comparison between survivors and non-survivors at diagnosis of acute respiratory distress syndrome

\begin{tabular}{|c|c|c|c|c|}
\hline \multirow{2}{*}{ 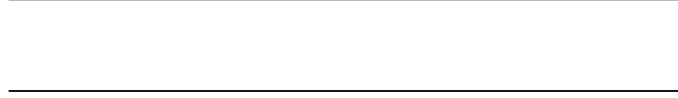 } & \multirow{2}{*}{$\begin{array}{l}\text { All patients } \\
n=442\end{array}$} & \multirow{2}{*}{$\begin{array}{l}\text { Survivor } \\
n=240\end{array}$} & \multirow{2}{*}{$\begin{array}{l}\text { Non-survivor } \\
n=202\end{array}$} & \multirow[t]{2}{*}{$p$-value } \\
\hline & & & & \\
\hline \multicolumn{5}{|l|}{ Basic characteristics } \\
\hline Age [years] & $50.0(37.0 ; 61.0)$ & $46.0(34.0 ; 60.0)$ & $53.0(42.0 ; 64.0)$ & $0.001^{*}$ \\
\hline Sex (male) & $285(64.5 \%)$ & $158(55.4 \%)$ & $127(44.6 \%)$ & 0.602 \\
\hline Weight [kg] & $80.0(70.0 ; 95.0)$ & $83.0(70.0 ; 100)$ & $80.0(69.5 ; 90.0)$ & $0.001^{*}$ \\
\hline Body mass index $\left[\mathrm{kg} / \mathrm{m}^{2}\right]$ & $28.4(8.46)$ & $29.6(8.81)$ & $26.8(7.73)$ & $0.001^{*}$ \\
\hline Transfer from other hospital & $307(69.5 \%)$ & $165(53.7 \%)$ & $142(46.3 \%)$ & 0.878 \\
\hline LOS hospital before admission [d] & $7.0(3.0 ; 15.0)$ & $6.0(3.0 ; 10.2)$ & $11.0(4.0 ; 23.0)$ & $<0.001^{*}$ \\
\hline LOS ICU before admission [d] & $4.0(2.0 ; 11.0)$ & $4.0(2.0 ; 7.0)$ & $6.0(2.0 ; 13.5)$ & $0.002^{*}$ \\
\hline Duration of mechanical ventilation before admission [d] & $3.0(2.0 ; 7.0)$ & $3.0(2.0 ; 6.0)$ & $4.0(2.0 ; 11.0)$ & $0.002^{*}$ \\
\hline \multicolumn{5}{|c|}{ Severity of illness, organ failure and comorbidities on ICU admission } \\
\hline APACHE ॥ & $28.0(20.0 ; 35.0)$ & $25.0(18.0 ; 35.2)$ & $29.0(24.0 ; 37.0)$ & $<0.001^{*}$ \\
\hline SAPS $\|$ & $54.0(39.0 ; 70.0)$ & $49.0(37.0 ; 63.0)$ & $61.0(42.5 ; 73.0)$ & $<0.001^{*}$ \\
\hline TISS & $50.0(43.0 ; 58.0)$ & $47.0(42.0 ; 56.0)$ & $53.0(45.0 ; 59.0)$ & $<0.001^{*}$ \\
\hline SOFA & $12.0(9.0 ; 15.0)$ & $11.0(9.0 ; 14.0)^{*}$ & $13.0(9.0 ; 16.0)^{*}$ & $<0.001^{*}$ \\
\hline $\mathrm{CCl}$ & $3.0(2.0-5.0)^{*}$ & $3.0(2.0-5.0)^{*}$ & $4.0(3.0-6.0)^{*}$ & $<0.001^{*}$ \\
\hline Aetiology of ARDS & & & & 0.137 \\
\hline - Pneumonia & $242(54,7 \%)$ & $133(55.0 \%)$ & $109(45.0 \%)$ & \\
\hline - Immuninsufficiency & $67(15.1 \%)$ & $30(44.8 \%)$ & $37(55.2 \%)$ & \\
\hline - Acute on chronic & $55(12.4 \%)$ & $26(47.3 \%)$ & $29(52,7 \%)$ & \\
\hline - Trauma & $29(6,6 \%)$ & $20(69.0 \%)$ & $9(31.0 \%)$ & \\
\hline - Sepsis of extrapulmonary origin & $27(6.1 \%)$ & $17(63.0 \%)$ & $10(37.0 \%)$ & \\
\hline - Other & $22(5.0 \%)$ & $14(63.3 \%)$ & $8(36.7 \%)$ & \\
\hline \multicolumn{5}{|l|}{ Severity of lung failure (assessed on day 1 ) } \\
\hline AECC Definition & & & & 0.170 \\
\hline ALI & $99(22.4 \%)$ & $60(60.6 \%)$ & $39(39.4 \%)$ & \\
\hline ARDS & $343(77.6 \%)$ & $180(52.5 \%)$ & $163(47.5 \%)$ & \\
\hline Berlin Definition & & & & 0.149 \\
\hline Mild & $99(22.4 \%)$ & $60(60.6 \%)$ & $39(39.4 \%)$ & \\
\hline Moderate & $210(47.5 \%)$ & $117(55.7 \%)$ & $93(44.3 \%)$ & \\
\hline Severe & $133(30.1 \%)$ & $63(47.4 \%)$ & $70(52.6 \%)$ & \\
\hline $\mathrm{PaO}_{2} / \mathrm{FiO}_{2}[\mathrm{mmHg}]$ & $137(92 ; 193)$ & $142(95 ; 199)$ & $123(89 ; 178)$ & $0.026^{*}$ \\
\hline Oxygenation index (OI) & $16.9(11.6 ; 27.4)$ & $16.4(11.1 ; 25.9)$ & $18.5(12.6 ; 28.2)$ & 0.063 \\
\hline Extracorporeal lung assist devices (ELAD) & & & & $<0.001^{*}$ \\
\hline No ELAD & $186(42.1 \%)$ & $130(69.9 \%)$ & $56(30.1 \%)$ & \\
\hline With ELAD & $256(57.9 \%)$ & $110(43.0 \%)$ & $146(57.0 \%)$ & \\
\hline - Only ECMO & $146(33.0 \%)$ & $61(41.8 \%)$ & $85(58.2 \%)$ & \\
\hline - Only ECLA & $74(16.7 \%)$ & $34(46.0 \%)$ & $40(54.0 \%)$ & \\
\hline$-\mathrm{ECLA}+\mathrm{ECMO}$ & $36(8.14 \%)$ & $15(41.7 \%)$ & $21(58.3 \%)$ & \\
\hline
\end{tabular}

Discrete variables are presented as number of percentage and were analysed with Chi square test for nonparametric samples. Continuous variables are presented as median and 25/75 percentiles and were analysed with Mann-Whitney-U-Test for nonparametric samples. ${ }^{*} p<0,05$

APACHE II Acute Physiology And Chronic Health Evaluation II, $d$ days, ICU intensive care unit, LOS length of stay, SAPS II Simplified Acute Physiology Score II, SOFA Sequential Organ Failure Assessment, TISS Therapeutic Intervention Scoring System, $\mathrm{CCl}$ Charlson comorbidity index, $\mathrm{F}_{i} \mathrm{O}_{2}$ inspiratory fraction of oxygen, iNO inhalative nitric oxide, $P B W$ predicted body weight, $P_{a} C_{2}$ arterial partial pressure of carbon dioxide, $P_{a} O_{2}$ arterial partial pressure of oxygen, $P E E P$ positive end-expiratory pressure, $P_{\text {mean }}$ mean airway pressure, $P_{\text {peak }}$ peak airway pressure, $V_{t}$ tidal volume, ECLA extracorporeal lung assist, ECMO extracorporeal membrane oxygenation, ELAD extracorporeal lung assist devices. Complete data on hospital stay in referring institutions was available for 309 patients 


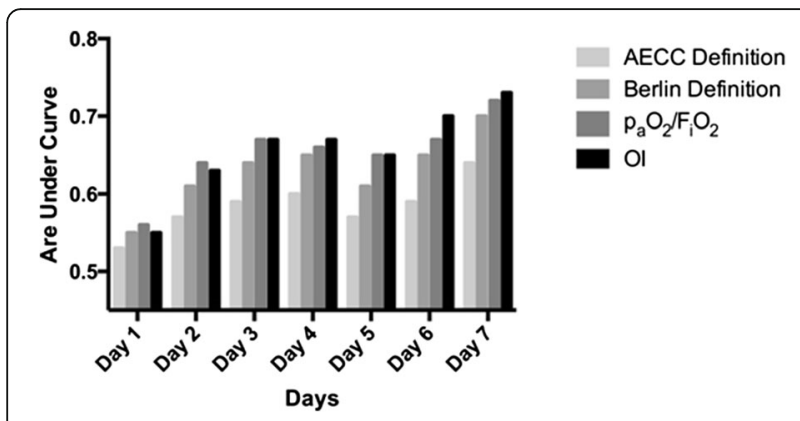

Fig. 1 Predicitive validity for in-hospital mortality for the first seven days of ARDS after its diagnosis for AECC and Berlin Definition of ARDS, $\mathrm{p}_{\mathrm{a}} \mathrm{O}_{2} / \mathrm{F}_{\mathrm{i}} \mathrm{O}_{2}$, and oxygenation index. Area under $\mathrm{ROC}$ curve shown for the first seven days of ARDS by 4 categorizing options of severity of lung failure: AECC and Berlin Definition of ARDS, $\mathrm{p}_{\mathrm{a}} \mathrm{O}_{2}$ / $\mathrm{F}_{\mathrm{i}} \mathrm{O}_{2}$ and $\mathrm{Ol}$
We suggest that application of our standard operating procedures for ARDS treatment reduced or even eliminated the influence of previous, different treatment approaches. Among the four evaluated criteria to classify or quantify severity of ARDS, OI was found to be the most accurate parameter with respect to predictive validity.

Regarding the time of assessment, the third day after admission to our referral centre was found to represent the best compromise between earliness and accuracy of prognosis of mortality in this patient group. In the group of patients with an OI of 15 or greater on day 3, mortality was higher; length of stay (both in the ICU and hospital) and duration of mechanical ventilation were longer. Furthermore, non-survivors had a significantly longer length of stay and duration of mechanical
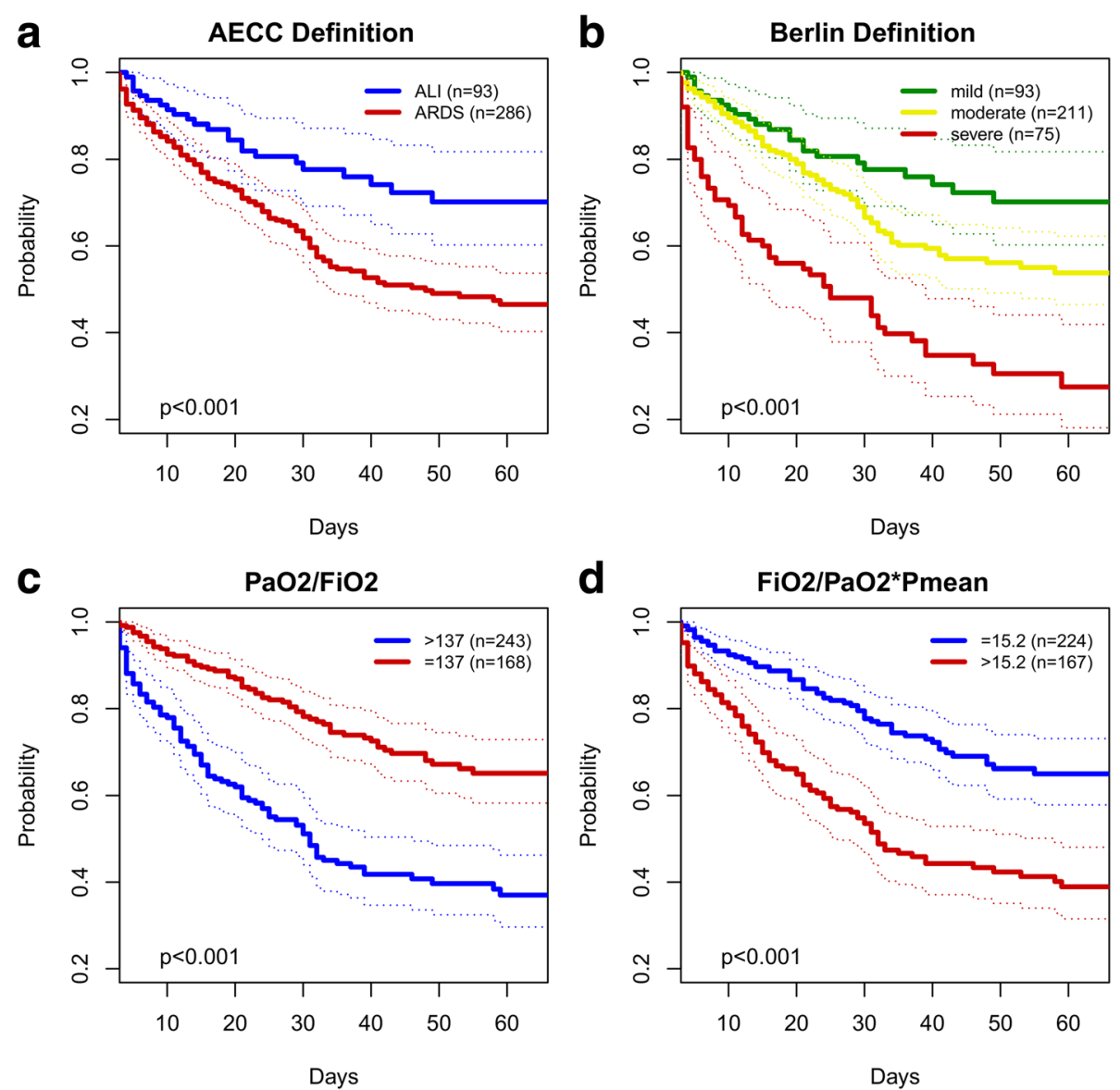

Fig. 2 Survival curves for $A E C C$ and Berlin definition of acute respiratory distress syndrome, $\mathrm{P}_{2} \mathrm{O}_{2} / \mathrm{F}_{1} \mathrm{O}_{2}$ ratio and Ol on day 3. Three hundred seventy nine patients had a $\mathrm{P}_{2} \mathrm{O}_{2} / \mathrm{F}_{i} \mathrm{O}_{2}$ ratio $\leq 300 \mathrm{mmHg}$ on day 3 and have been grouped in the corresponding stages of the AECC (a) and Berlin definition (b). In total, $\mathrm{P}_{\mathrm{a}} \mathrm{O}_{2} / \mathrm{F}_{\mathrm{i}} \mathrm{O}_{2}$ was available for all 411 patients alive on day 3 (c). Values for $\mathrm{F}_{\mathrm{i}} \mathrm{O}_{2} / \mathrm{P}_{\mathrm{a}} \mathrm{O}_{2}{ }^{*} \mathrm{P}_{\text {mean }}$ were available for 391 patients being mechanically ventilated on that day (d). AECC: American-European Consensus Conference; $F_{i} \mathrm{O}_{2}$ : inspiratory fraction of oxygen; $F_{i} \mathrm{O}_{2} /$ $\mathrm{P}_{a} \mathrm{O}_{2}{ }^{*} P_{\text {mean }}$ describes oxygenation index. $\mathrm{P}_{a} \mathrm{O}_{2}$ : arterial partial pressure of oxygen; $P_{\text {mean }}$ : mean airway pressure, $\mathrm{O}$ : oxygenation index 
Table 2 Multivariate Cox regression on factors influencing hospital mortality

\begin{tabular}{llll}
\hline & $p$-value & $\mathrm{HR}$ & $95 \% \mathrm{Cl}$ \\
\hline $\mathrm{CCl}$ & $0.023^{*}$ & 1.08 & $1.010-1.149$ \\
Ol on day 3 (per point) & $<0.001^{*}$ & 1.03 & $1.015-1.047$ \\
Age (per year) & $0.003^{*}$ & 1.02 & $1.006-1.029$ \\
TISS28 on admission & $0.006^{*}$ & 1.02 & $1.007-1.043$ \\
Crs $_{\text {On day 3 }}$ & $0.003^{*}$ & 0.98 & $0.971-0.994$ \\
Prone position on day 3 & $0.002^{*}$ & 0.81 & $0.711-0.928$ \\
BMI & 0.097 & 0.98 & $0.956-1.004$ \\
pH on day 3 & $0.002^{*}$ & 0.03 & $0.002-0.275$ \\
\hline
\end{tabular}

Parameters considered in the multivariate regression model using backwards selection that had significant impact in univariate analyses: age, BMI, Charlson comorbidity index (CCI), ICU admission scores (APACHE II, SOFA, TISS28), ELAD (yes/no) as well as clinical variables that were assessed on day 3 (SOFA score), $C_{r s}$ Compliance of respiratory system by $C_{r s}=V_{T} /\left(P_{\text {plat }}-P_{\text {mean }}\right) p H$ day $3, V_{t} /$ PBW, $P_{\text {peak, }}$ Prone position, categories of Berlin definition and AECC definition, $\mathrm{P}_{\mathrm{a}} \mathrm{O}_{2} / \mathrm{F}_{\mathrm{i}} \mathrm{O}_{2}$. Data of 341 patients were considered. ${ }^{*} p<0,05$. All parameters of the final regression model are presented, regardless of their significance $B M I$ Body Mass Index, CCI Charlson comorbidity index, Cl confidence interval, $E L A D$ extracorporeal lung assist devices (including extracorporeal lung assist (ECLA) and extracorporeal membrane oxygenation (ECMO), $F_{i} \mathrm{O}_{2}$ inspiratory fraction of oxygen, $H R$ Hazard Ratio, Ol oxygenation index, $P B W$ predicted body weight, $P_{\text {peak }}$ peak airway pressure, TISS Therapeutic Intervention Scoring System

ventilation in referring hospitals before being admitted to the ICU than survivors of ARDS.

The OI was originally designed as a predictive tool for pediatric patients with hypoxemic conditions [13, 14]. Later, this factor has been taken into consideration in adults suffering from ARDS $[15,16]$. Our findings regarding compromised outcome in patients with an OI of 15 or greater are in line with previous findings indicating that the $\mathrm{OI}$ is equivalent to or even better than other mortality prediction parameters used for ARDS, and our study further substantiated this finding. We propose that the OI might be one of the preferable predictive parameters because it exclusively accounts for changes in mean airway pressure and thereby reflects invasiveness of mechanical ventilation to some extent. However, most large observational studies on predictive parameters in ARDS did not assess the OI to adjust the oxygenation ration ratio to the invasiveness of ventilation or did not report it [17, 18]. Moreover, currently used categorizing systems, i.e. the AECC and the Berlin definition of ARDS, do not consider the OI in their panels of defining variables $[1,3]$. To include invasiveness of ventilation, a PEEP level of more or equal than five $\mathrm{cm} \mathrm{H}_{2} \mathrm{O}$ became part of the definition. In contrast, the mean PEEP level in our study group was $17 \mathrm{~cm} \mathrm{H}_{2} \mathrm{O}$, which demonstrates the severity of illness in our patient population.

In contrast to OI, the $\mathrm{P}_{\mathrm{a}} \mathrm{O}_{2} / \mathrm{F}_{\mathrm{i}} \mathrm{O}_{2}$ ratio was not an independent predictor of mortality in our study. Although this parameter is often used to describe oxygenation status in critically ill patients, it also failed to predict clinical outcome at the onset of ARDS in recent studies $[19,20]$. In this respect, our data support the concept that the $\mathrm{P}_{\mathrm{a}} \mathrm{O}_{2} / \mathrm{F}_{\mathrm{i}} \mathrm{O}_{2}$ ratio alone might not be suitable to determine clinical outcome in ARDS. One explanation might be that the $\mathrm{P}_{\mathrm{a}} \mathrm{O}_{2} / \mathrm{F}_{\mathrm{i}} \mathrm{O}_{2}$ ratio is a highly variable index depending on ventilator settings, conditions of patients and, to a more or lesser extend, routinely performed therapeutic interventions such as bronchoscopies or positioning. $\mathrm{F}_{\mathrm{i}} \mathrm{O}_{2}$ by itself, independently of $\mathrm{P}_{\mathrm{a}} \mathrm{O}_{2} / \mathrm{F}_{\mathrm{i}} \mathrm{O}_{2}$, is usually not considered to select patients regarding their risk of poor outcome in larger clinical trails. However, it has been shown that after controlling for baseline $\mathrm{P}_{\mathrm{a}} \mathrm{O}_{2} / \mathrm{F}_{\mathrm{i}} \mathrm{O}_{2}$, $\mathrm{F}_{\mathrm{i}} \mathrm{O}_{2}$ was able to predict mortality [21].

Villar and colleagues demonstrated that the $\mathrm{P}_{\mathrm{a}} \mathrm{O}_{2} / \mathrm{F}_{\mathrm{i}} \mathrm{O}_{2}$ ratio obtained $24 \mathrm{~h}$ after ARDS onset allowed a better risk classification when $\mathrm{F}_{\mathrm{i}} \mathrm{O}_{2}$ was at least 0.5 with a PEEP of at least $10 \mathrm{~cm} \mathrm{H}_{2} \mathrm{O}$ [22]. The OI was, however, not part of the Villar study. A side by side comparison in our study of the four different parameters on day 3 showed that the OI was the only parameter that was

Table 3 Clinical outcome parameters grouped by cut-off (15.2) of the oxygenation index $\mathrm{F}_{\mathrm{i}} \mathrm{O}_{2} / \mathrm{P}_{\mathrm{a}} \mathrm{O}_{2}{ }^{*} \mathrm{P}_{\text {mean }}(\mathrm{Ol})$ on day 3

\begin{tabular}{llll}
\hline & $\begin{array}{l}\text { All patients } \\
n=442\end{array}$ & $\begin{array}{l}\text { Ol day } 3<15 \\
n=242\end{array}$ & $\begin{array}{l}\text { Ol day } 3 \geq 15 \\
n=167\end{array}$ \\
\hline $\begin{array}{l}\text { Type of discharge } \\
\text { - Deceased }\end{array}$ & $202(45.7 \%)$ & $71(29.3 \%)$ & $102(61.1 \%)$ \\
- Discharged to home & $60(13.6 \%)$ & $40(16.5 \%)$ & $17(10.2 \%)$ \\
- Transfer to another hospital & $95(21.5 \%)$ & $54(22.3 \%)$ & $27(16.2 \%)$ \\
- Transfer to rehabilitation facility & $85(19.2 \%)$ & $59(24.3 \%)$ & $21(12.6 \%)$ \\
LOS ICU [d] & $36.0(25.0 ; 57.0)$ & $33.5,(21.8 ; 53.0)$ & $43.0(31.0 ; 61.0)$ \\
LOS hospital [d] & $43.0(27.8 ; 66.0)$ & $41.0(24.0 ; 64.0)$ & $48.0(34.0 ; 72.0)$
\end{tabular}

Discrete variables are presented as median and percentage and were analysed with Chi square test for nonparametric samples. Continuous variables are presented as median and 25/75 percentiles and were analysed with Mann-Whitney-U-Test for nonparametric samples. ${ }^{*} p<0,05$

$d$ days, $h$ hours, ICU intensive care unit, $n$ number, $L O S$ length of stay, $O I$ oxygenation index calculated as median for each day by $\mathrm{OI}=\left(\mathrm{F}_{i} \mathrm{O}_{2} / \mathrm{P}_{\mathrm{a}} \mathrm{O}_{2}[\mathrm{mmHg}]\right) \times$ $\left.P_{\text {mean }} \times 100\right)$. The cut-off value was derived from Youden - Index corresponding to the area under the ROC curve for OI on day 3 for all patients 
capable of predicting mortality in multivariate analyses. The other three, including the $\mathrm{P}_{\mathrm{a}} \mathrm{O}_{2} / \mathrm{F}_{\mathrm{i}} \mathrm{O}_{2}$ ratio, were not identified as independent predictors.

As a known fact, PEEP was not part of the AECC definition but was included in the Berlin definition for classifying ARDS. More studies were conducted to verify the predictive properties of PEEP in the definition of ARDS after the publications of AECC and Berlin guidelines. Since the Berlin definition defines the minimum of PEEP to be $5 \mathrm{~cm} \mathrm{H}_{2} \mathrm{O}$, many studies followed this assumption and concluded differing results. As an example, when Britos and coworkers categorized their cases as PEEP $<5 \mathrm{~cm} \mathrm{H}_{2} \mathrm{O}(1.3 \%)$ and $\geq 10 \mathrm{~cm} \mathrm{H}_{2} \mathrm{O}$ (50\%), they found that after adjusting for $\mathrm{P}_{\mathrm{a}} \mathrm{O}_{2} / \mathrm{F}_{\mathrm{i}} \mathrm{O}_{2}$ baseline PEEP did not predict mortality [21]. In contrast, in our study the median PEEP was $17 \mathrm{~cm} \mathrm{H}_{2} \mathrm{O}$, which may be one of the conditions facilitating the finding of a predictable parameter when requiring a higher PEEP. Goligher and coworkers observed in a patient group with a median PEEP of $9.5 \mathrm{~cm} \mathrm{H}_{2} \mathrm{O}$, that positive oxygenation response to PEEP elevation may predict mortality [23]. Golighers conclusions may not be applied to our patient population that was treated with median PEEP of $17 \mathrm{~cm} \mathrm{H}_{2} \mathrm{O}$. In this regard, the use of a PEEP level of $5 \mathrm{~cm} \mathrm{H}_{2} \mathrm{O}$ recommended by the Berlin definition might not be appropriate to discriminate the severity of illness and does not allow reliable mortality prediction, respectively. Furthermore, if a PEEP level of $5 \mathrm{~cm} \mathrm{H}_{2} \mathrm{O}$ would have been applied in our patient population, it is strongly expected that the number of severe ARDS cases would increase. Hence, the number of severe cases reported in this manuscript is supposedly underestimated from this perspective. In line with aforementioned studies, this observation underlies the importance of standardized conditions for ARDS classification in order to render investigations from different settings comparable.

In our study, the third day of $\mathrm{P}_{\mathrm{a}} \mathrm{O}_{2} / \mathrm{F}_{\mathrm{i}} \mathrm{O}_{2}<300 \mathrm{mmHg}$ in our centre was found to represent the best compromise between earliness and accuracy of prognosis of mortality in this patient group. As a special referral centre we are sometimes unable to identify the exact onset of ARDS, which is a limitation of our study. We suggest that the very different treatment approaches in pretreating hospitals bias comparability of patients at the time of referral to our centre. In the study by Peek and colleagues, the different treatment procedures in hospitals other than ECMO centres were considered as a major limitation when comparing patients [24]. In our opinion, algorithm-guided -and most importantly homogeneous standard treatment approaches including ventilator settings, inhalation of nitric oxide, prone positioning or volume therapy in an ARDS centre may eliminate these confounding effects. In this context, our data suggests that length of stay in hospitals before transfer to a specialized ARDS treatment centre could impact mortality.

\section{Conclusions}

The Berlin definition has marked a major step for a uniform classification of ARDS. As major finding of our study, the OI has been proven to be a more suitable parameter to predict mortality of patients suffering from ARDS in national referral centres when compared to $\mathrm{P}_{\mathrm{a}} \mathrm{O}_{2} / \mathrm{F}_{\mathrm{i}} \mathrm{O}_{2}$ ratio, the AECC or Berlin definition. The OI appears to be especially helpful for prediction of mortality on day three after admission to a specialized referral centre, as a response to standardised ARDS treatment may be observed by that time. Secondly, early patient transfers to specialized ARDS centres are associated with increased survival.

\section{Additional files}

Additional file 1: Table S1. Selected patient-specific parameters on day 1 and day 3. (DOC 49 kb)

Additional file 2: Table S3. Number of patients in prone position on each day. IQR: $1 \mathrm{~d}-2 \mathrm{~d}$. (DOC $25 \mathrm{~kb}$ )

Additional file 3: Table S4. Number of patients receiving extracorporeal lung assist devices (ELAD) on each day. (DOC 26 kb)

Additional file 4: Figure S1. Predictive validity of oxygenation index for in-hospital mortality for the first seven days of ARDS grouped by need of extracorporeal lung assist devices (ELAD). (JPG 38 kb)

Additional file 5: Table S2. Full model of multivariate Cox regression analysis on factors influencing hospital mortality. (DOC $37 \mathrm{~kb}$ )

\section{Abbreviations}

AECC: American European consensus conference on ARDS; ALI: Acute lung injury; APACHE II: Acute physiology and chronic health evaluation II; ARDS: Acute respiratory distress syndrome; AUC: Area under curve; CCl: Charlson comorbidity index; COPD: Chronic obstructive pulmonary disease; ECLA: Extracorporeal lung assist; ECMO: Extracorporeal membrane oxygenation; ELAD: Extracorporeal lung assistant devices; $\mathrm{F}_{\mathrm{j}} \mathrm{O}_{2}$ : Fraction of inspired oxygen; ICU: Intensive care unit; Ol: Oxygenation index; $\mathrm{P}_{2} \mathrm{O}_{2}$ : Arterial partial pressure of oxygen; PBW: Predicted body weight; PEEP: Positive endexspiratory pressure; SAPS II: Simplified acute physiology score; SOFA: Sequential organ failure assessment; $V_{\mathrm{T}}$ : Tidal volume

\section{Acknowledgements}

The authors would like to thank Eike Kühn for his excellent help in data preparation and processing.

\section{Funding}

No external funding has been obtained for this study.

\section{Availability of data and materials}

The datasets analysed during the current study are available from the corresponding author on reasonable request.

\section{Authors' contributions}

FB performed data collection and statistical analysis; drafted parts of the manuscript; shared responsibility for design, coordination, and finalization of the manuscript; contributed to interpretation of the data, and had full access to the data. MM drafted the introduction and discussion section. JZ performed data collection and contributed to the draft of all parts of the manuscript. KW contributed to the design of the study regarding statistical analysis, interpretation of the data, was involved in drafting the methods section, and critically reviewed the manuscript. MM, CP, CS, and MS 
contributed to the draft of the discussion section and critically revised the manuscript for intellectual content. SW and MD outlined the study design; contributed to draft all sections of the manuscript, interpretation of data, shared responsibility for design, coordination, and finalization of the manuscript; and had full access to the data. All authors read and approved the manuscript.

\section{Competing interests}

The authors declare that they have no competing interests regarding the submitted work. Claudia Spies received funding unrelated to this study from Abbott, Aspect, Baxter, BBraun, Essex Pharma, Fresenius Kabi, GSK, and Wyeth. Steffen Weber-Carstens received honoraria for lectures from Dräger, Maquet, and Novalung.

\section{Consent for publication}

Not applicable.

\section{Ethics approval and consent to participate}

The study was approved by the written consent of the Ethics Commission Charité - Universitätsmedizin Berlin (EA1/223/12). The need for patient's consent was waived due to the retrospective nature of the study.

\section{Author details}

'Department of Anesthesiology and Intensive Care Medicine, Charité Universitätsmedizin Berlin, Campus Virchow-Klinikum / Campus Charité Mitte, Augustenburger Platz 1, D-13353 Berlin, Germany. ${ }^{2}$ Department of Anesthesiology and Intensive Care Medicine, Charité - Universitätsmedizin Berlin, Campus Benjamin Franklin, Hindenburgdamm 30, 12203 Berlin, Germany. ${ }^{3}$ SOSTANA GmbH, Wildensteiner Str. 27, 10318 Berlin, Germany. ${ }^{4}$ Department of Anaesthesiology and Intensive Care Medicine, Werner Forßmann Krankenhaus, 16225 Eberswalde, Germany.

\section{Received: 20 October 2015 Accepted: 14 October 2016}

\section{Published online: 08 November 2016}

\section{References}

1. Bernard GR, Artigas A, Brigham KL, Carlet J, Falke K, Hudson L, Lamy M, Legall JR, Morris A, Spragg R. The American-European Consensus Conference on ARDS. Definitions, mechanisms, relevant outcomes, and clinical trial coordination. Am J Respir Crit Care Med. 1994;149(3 Pt 1):818-24.

2. Phua J, Stewart TE, Ferguson ND. Acute respiratory distress syndrome 40 years later: time to revisit its definition. Crit Care Med. 2008;36:2912-21.

3. Ranieri VM, Rubenfeld GD, Thompson BT, Ferguson ND, Caldwell E, Fan E, Camporota L, Slutsky AS. Acute respiratory distress syndrome: the Berlin Definition. J Am Med Assoc. 2012;307:2526-33

4. Dechert RE, Park PK, Bartlett RH. Evaluation of the oxygenation index in adult respiratory failure. J Trauma Acute Care Surg. 2014;76:469-73.

5. Kao H-C, Lai T-Y, Hung H-L, Chen Y-M, Chou P-A, Wang C-C, Lin M-C, Fang $W$-F. Sequential oxygenation index and organ dysfunction assessment within the first 3 days of mechanical ventilation predict the outcome of adult patients with severe acute respiratory failure. Sc World J. 2013;2013:413216.

6. Villar J, Pérez-Méndez L, Basaldúa S, Blanco J, Aguilar G, Toral D, Zavala E, Romera MA, González-Díaz G, Nogal FD, Santos-Bouza A, Ramos L, Macías S, Kacmarek RM. A risk tertiles model for predicting mortality in patients with acute respiratory distress syndrome: age, plateau pressure, and $\mathrm{P}(\mathrm{aO}(2))$ / $\mathrm{F}(\mathrm{IO}(2))$ at ARDS onset can predict mortality. Respir Care. 2011:56:420-8.

7. Spies C, Kastrup M, Kerner T, Melzer-Gartzke C, Zielke H. SOPs in Intensivmedizin Und Notfallmedizin: Alle Relevanten Standards Und Techniken Für Die Klinik. 1st ed. Stuttgart: Thieme; 2013.

8. Deja M, Hommel M, Weber-Carstens S, Moss M, von Dossow V, Sander M, Pille C, Spies C. Evidence-based therapy of severe acute respiratory distress syndrome: an algorithm-guided approach. J Int Med Res. 2008;36:211-21.

9. Charlson ME, Pompei P, Ales KL, MacKenzie CR. A new method of classifying prognostic comorbidity in longitudinal studies: development and validation. J Chronic Dis. 1987:40:373-83.

10. Brun-Buisson C, Minelli C, Bertolini G, Brazzi L, Pimentel J, Lewandowski K, Bion J, Romand J-A, Villar J, Thorsteinsson A, Damas P, Armaganidis A, Lemaire F. Epidemiology and outcome of acute lung injury in European intensive care units. Results from the ALIVE study. Intensive Care Med. 2004;30:51-61.
11. Wohlfarth $P$, Ullrich $R$, Staudinger $T$, Bojic A, Robak O, Hermann A, Lubsczyk B, Worel N, Fuhrmann V, Schoder M, Funovics M, Rabitsch W, Knoebl P, Laczika K, Locker GJ, Sperr WR, Schellongowski P. Extracorporeal membrane oxygenation in adult patients with hematologic malignancies and severe acute respiratory failure. Crit Care Lond Engl. 2014;18:R20.

12. Fluss $R$, Faraggi $D$, Reiser $B$. Estimation of the Youden Index and its associated cutoff point. Biom J Biom Z. 2005;47:458-72.

13. Heiss KF, Bartlett RH. Extracorporeal membrane oxygenation: an experimental protocol becomes a clinical service. Adv Pediatr. 1989:36:117-35.

14. Durand M, Snyder JR, Gangitano E, Wu PY. Oxygenation index in patients with meconium aspiration: conventional and extracorporeal membrane oxygenation therapy. Crit Care Med. 1990;18:373-7.

15. Monchi M, Bellenfant F, Cariou A, Joly LM, Thebert D, Laurent I, Dhainaut JF, Brunet F. Early predictive factors of survival in the acute respiratory distress syndrome. A multivariate analysis. Am J Respir Crit Care Med. 1998;158:1076-81.

16. Gajic O, Afessa B, Thompson BT, Frutos-Vivar F, Malinchoc M, Rubenfeld GD, Esteban A, Anzueto A, Hubmayr RD. Prediction of death and prolonged mechanical ventilation in acute lung injury. Crit Care Lond Engl. 2007:11:R53.

17. Brower RG, Lanken PN, Maclntyre N, Matthay MA, Morris A, Ancukiewicz M, Schoenfeld D, Thompson BT. Higher versus lower positive end-expiratory pressures in patients with the acute respiratory distress syndrome. N Engl J Med. 2004;351:327-36

18. Brower RG, Matthay MA, Morris A, Schoenfeld D, Thompson BT, Wheeler A, Wiedemann HP, Arroliga AC, Fisher CJ, Komara JJ, et al. Ventilation with lower tidal volumes as compared with traditional tidal volumes for acute lung injury and the acute respiratory distress syndrome. N Engl J Med. 2000;342:1301-8

19. Miles LF, Bailey M, Young P, Pilcher DV. Differences in mortality based on worsening ratio of partial pressure of oxygen to fraction of inspired oxygen corrected for immune system status and respiratory support. Crit Care Resusc J Australas Acad Crit Care Med. 2012;14:25-32.

20. Ware LB. Prognostic determinants of acute respiratory distress syndrome in adults: impact on clinical trial design. Crit Care Med. 2005;33(3 Suppl):S217-22.

21. Britos M, Smoot E, Liu KD, Thompson BT, Checkley W, Brower RG. The value of positive end-expiratory pressure and $\mathrm{FiO}_{2}$ criteria in the definition of the acute respiratory distress syndrome. Crit Care Med. 2011:39:2025-30.

22. Villar J, Blanco J, Añón JM, Santos-Bouza A, Blanch L, Ambrós A, Gandía F, Carriedo D, Mosteiro F, Basaldúa S, Fernández RL, Kacmarek RM. The ALIEN study: incidence and outcome of acute respiratory distress syndrome in the era of lung protective ventilation. Intensive Care Med. 2011;37:1932-41.

23. Goligher EC, Kavanagh BP, Rubenfeld GD, Adhikari NKJ, Pinto R, Fan E, Brochard L, Granton JT, Mercat A, Marie Richard J-C, Chretien J-M, Jones GL, Cook DJ, Stewart TE, Slutsky AS, Meade MO, Ferguson ND. Oxygenation response to positive end-expiratory pressure predicts mortality in acute respiratory distress syndrome. A secondary analysis of the LOVS and ExPress trials. Am J Respir Crit Care Med. 2014;190:70-6.

24. Peek GJ, Mugford M, Tiruvoipati R, Wilson A, Allen E, Thalanany MM, Hibbert CL, Truesdale A, Clemens F, Cooper N, Firmin RK, Elbourne D. Efficacy and economic assessment of conventional ventilatory support versus extracorporeal membrane oxygenation for severe adult respiratory failure (CESAR): a multicentre randomised controlled trial. Lancet. 2009:374:1351-63.

\section{Submit your next manuscript to BioMed Central and we will help you at every step:}

- We accept pre-submission inquiries

- Our selector tool helps you to find the most relevant journal

- We provide round the clock customer support

- Convenient online submission

- Thorough peer review

- Inclusion in PubMed and all major indexing services

- Maximum visibility for your research

Submit your manuscript at www.biomedcentral.com/submit
Biomed Central 